\title{
Industrial experiment on electrodialized separation of highly concentrated multicomponent technological solutions at thermal power plants
}

\author{
A. A. Filimonova ${ }^{1}$, N. D. Chichirova ${ }^{1}$, A. A. Chichirov ${ }^{1, *}$, and A. I. Minibaev ${ }^{1}$ \\ ${ }^{1}$ Kazan State Power Engineering University, Kazan, Russia
}

\begin{abstract}
The main sources of highly concentrated multicomponent technological solutions at thermal power plants (TPPs) are water treatment plants. Analysis of operation of the ion-exchange water treatment plant at the Nizhnekamsk Thermal Power Plant-1 showed that half of alkali supplied to regeneration of the anion-exchange alkali filters is not used, but is discharged for neutralization and then to wastewater. Due to the fact that the cost of alkali used in technological processes is quite high, it is economically feasible to process the alkaline waste with the alkali extraction and its reuse in the production cycle. The article presents the experimental results on the electro-membrane separation of alkaline waste regeneration solutions and wash water after anion-exchange filter regeneration. The revealed differences in the selectivity of various ion transfer through the electrodialysis apparatus membranes, depending on time and amount of transmitted electricity, allowed us to establish the possibility of obtaining an alkaline solution purified from impurities.
\end{abstract}

\section{Introduction}

Thermal power plants (TPPs) in Russia use electrochemical methods of water treatment in a small volume and only as additional methods of water purification [1-4]. On the other hand, in the Soviet Union scientific research was actively conducted in this area and scientific and technological base was developed for the industrial use of electrodialysis at thermal power plants. In 1970-80, the accumulated results concerning usage of electro-membrane plants with partial and deep water desalination were generalized, electro-membrane schemes for concentrating of the spent regeneration water solutions were tested, the possibilities of using electrodialysis to produce acid and alkali from saline wastewater were investigated.

Electrodialysis projects for waste disposal are being actively developed worldwide [5-11]. The lack of effective technical solutions for the ballast salts processing into valuable products also make it a significant amount of high-salt waste that is problematic for further treatment. Electrochemical methods are effective tools and need low-duty conditions for concentration, separation, and purification of highly concentrated solutions of complex compositions containing a mixture of organic and inorganic substances. The development and application of these technologies is increasingly contributing to their sustainable use in various areas of bio-food industry, biotechnology, pharmaceuticals, water treatment at power plants, wastewater treatment [12-16].

The main advantage of electrochemical, and especially electro-membrane methods, is that chemical reactions and transformations are conducted using electric current, which are impossible or very difficult to perform by other means. This advantage can and should be used in various industries not only for purification of aqueous solutions, but also for separation of multicomponent complex mixtures into individual substances suitable for subsequent return to the production or reuse cycle.

From 2014 to present, a pilot industrial plant for electro-membrane utilization of purge water from an evaporative water treatment plant with an alkaline and softened saline has been successfully operated at Kazan TPP-3 [17]. The idea of purge water separation into alkaline and softened solutions and their use in the TPP cycle was formulated in connection with a high content of alkali and alkaline components (sodium carbonate) in the purge. Alkali and soda are expensive products that are widely used in water treatment plants of thermal power plants.

Due to the prospects of electrochemical processing of complex composition concentrated solutions, the goal was set to separate alkaline waste regeneration solutions (AWRS) and wash water after regeneration of anionite filters of a water treatment plant (WTP) using electromembrane technologies at Nizhnekamsk TPP -1 .

\footnotetext{
*Corresponding author: aachichirova@mail.ru
} 
Table 1. Characterization of AWRS fractions from anionite semi-chains of water treatment plant. $\mathrm{A}(\mathrm{ph} / \mathrm{ph})$ is alkalinity for phenolphthalein, SEC is electrical conductivity, $\mathrm{PO}$ is permanganate oxidation.

\begin{tabular}{|c|c|c|c|c|c|c|}
\hline Fraction & Volume, tons & Color & $\mathbf{p H}$ & $\mathbf{A}(\mathbf{p h} / \mathbf{p h}), \mathbf{m o l}-\mathbf{e q} / \mathbf{l}$ & $\mathbf{S E C}, \mathbf{m S} / \mathbf{c m}$ & $\mathbf{P O}, \mathbf{m g O} / \mathbf{l}$ \\
\hline 1 & 20 & clear & 7.2 & 0 & 1 & 80.4 \\
\hline 2 & $30-35$ & light yellow & 8 & 0.0004 & 39 & 128 \\
\hline 3 & 20 & black-brown & 12.82 & 0.4 & 120 & 384 \\
\hline 4 & 20 & yellow & & 0.212 & 55.1 & 100 \\
\hline 5 & 20 & light yellow & 12.34 & 0.02 & 5.2 & 5 \\
\hline
\end{tabular}

\section{Methods}

To determine the possibility of alkaline solution separation and its reuse in the station cycle, we performed analysis of the anionite filter regenerating technology and determination of the qualitative and quantitative composition of AWRS.

At the chemical desalting WTP of the Nizhnekamsk TPP-1, regeneration of anion-exchange filters is carried out according to a block scheme with $2-3 \%$ alkali firstly passing through the second stage filter (AnII) and then through the first one (AnI). All AWRS water and most of wash water are discharged to neutralize. The remaining part of wash water (having alkalinity less than $200 \mu \mathrm{g}-\mathrm{eq} / \mathrm{l})$ is collected in an auxiliary tank for reuse, such as anionite filter loosening. The filters are washed off with decarbonated water.

Water during the regeneration of anion "semi-chains" exchangers comes out with AnI in the form of individual fractions that are almost equal in volume to a characteristic chemical composition (table 1).

During the regeneration, the practically pure, neutral water (fraction 1, "pure" water) is washed away from the first 20 tons. These are the remnants of wash water being forced out of the filters. Further, 30-35 tons of neutral saline are released. These are mainly sodium salts of strong acids (sulfuric and hydrochloric) with silicic acid admixtures (fraction 2, a neutral "soft" solution). Next, the output is determined by a black solution with an alkaline $\mathrm{pH}$ that is contaminated with impurities "removed" from the filters, i.e. strong acid anions and organic substances (fraction 3, "dirty alkali"). After that, the impurity content decreases sharply, the fraction of "conditionally clean" alkali begins to come out (fraction 4). The alkali proportion in fraction No. 4 is $30 \%$ of the alkali initial amount supplied for regeneration, and the concentration of impurities in it is not more than $5 \%$ mass. The last fraction No. 5 is alkaline wash water with a low content of impurities and alkali.

$6 \pm 3 \%$ of the initial alkali amount supplied for regeneration is spent on regeneration of AnII filters. The main alkali amount (up to 50\%) is spent on regeneration of the AnI filters.

Thus, the rate of usage of alkali directly for regeneration is slightly more than $50 \%$, and the losses sent for neutralization is about $50 \%$ of the total consumption for regeneration. Based on this calculation, the expediency of the work goal is substantiated.

\section{Results and discussion}

Field tests were carried out using the experimental electro-membrane installation EMA 200/2T, having 200 chambers, with cation- and anion-exchange membrane alternation.

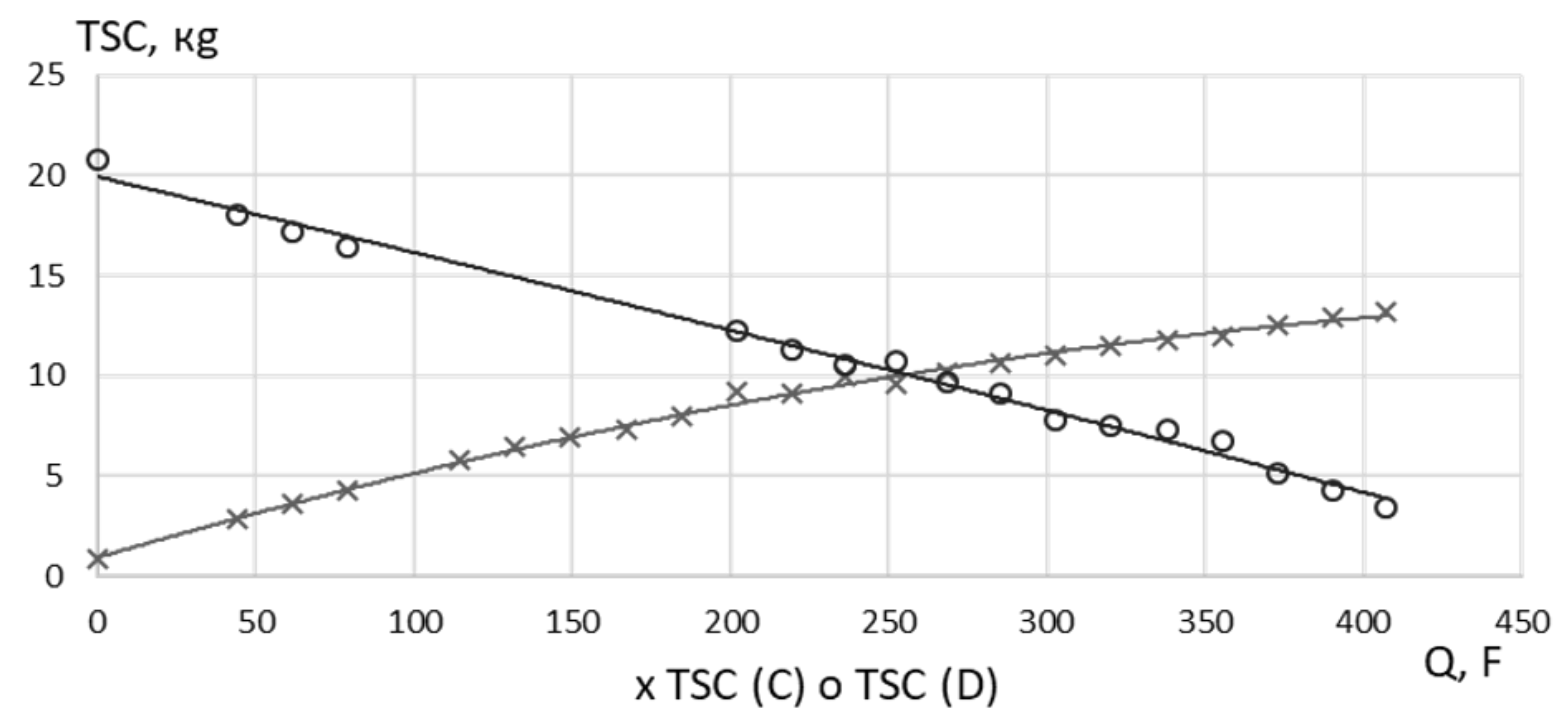

Figure 1. Dynamics of TSC transfer at a current load of $28.3 \mathrm{~A}$. The volume and composition of the concentrate is 2701 of partially desalinated water, of dialysate it is 7501 of fraction 3 of AWRS. 
Table 2. Calculated data on the separation ratio and selectivity of industrial water component transfer of industrial waters (fraction No. 4) through a membrane pair in an EMA. Cc is the concentration of the analyzed component in the concentrate after electrodialysis, $\mathrm{Cd}$ is the concentration of the analyzed component in dialysate after electrodialysis, Cdin is the concentration of the analyzed component in dialysate before electrodialysis.

\begin{tabular}{|c|c|c|c|c|}
\hline \multirow[b]{2}{*}{ Index } & \multicolumn{3}{|c|}{ Ratio } & \multirow[b]{2}{*}{$\begin{array}{c}\text { Specific energy } \\
\text { consumption of dialysis } \\
\text { (mol-eq/F per } 1 \text { hour) }\end{array}$} \\
\hline & $\begin{array}{l}\text { Separation, Rs = } \\
\text { Cc/Cd after } 1.5 \\
\text { hours of ED }\end{array}$ & $\begin{array}{c}\text { Concentration, } \\
\mathrm{Rc}=\mathrm{Cc} / \mathrm{Cd}_{\text {in }} \text { after } 1.5 \\
\text { hours of } \mathrm{ED}\end{array}$ & $\begin{array}{c}\text { Reduce, } \\
\mathrm{Rr}=\mathrm{Cd}_{\text {in }} / \mathrm{Cd} \text { after } 1.5 \\
\text { hours of } \mathrm{ED}\end{array}$ & \\
\hline TSC & 10.4 & 3.09 & 3.38 & \\
\hline $\mathrm{Na}^{+}$ & 9.4 & 2.87 & 3.27 & 0.76 \\
\hline $\mathrm{A}(\mathrm{ph} / \mathrm{ph})$ & 8.5 & 2.85 & 3 & 0.76 \\
\hline $\mathrm{Cl}^{-}$ & 3.45 & 1.5 & 2.28 & 0.23 \\
\hline $\mathrm{SO}_{4}{ }^{2-}$ & 1.65 & 1.3 & 1.25 & 0.012 \\
\hline $\mathrm{SiO}_{3}{ }^{2-}$ & 1.25 & 0.62 & 2.02 & 0.004 \\
\hline organic matter & 0.59 & 0.47 & 1.24 & 0.0012 \\
\hline $\mathrm{Fe}^{2+}$ & 0.6 & 0.52 & 1.15 & 0.0006 \\
\hline
\end{tabular}

According to the results of the AWRS fraction analyzes, it was decided to separate the most concentrated alkaline 3 and 4 fractions (Table 1). During the process, the ions move under the action of electric current through the membranes from the dialysate to the concentrate. The dynamics of concentration changes depending on the electricity amount passed through the solutions is shown in Figure 1. This is the electrolyte matter transfer, determined through the total salt content (TSC), from the dialysate line and the TSC accumulation in the concentrate line. The TSC change was calculated through the specific electrical conductivity of solutions. By the nature of curves in the graph, one can describe the process of substance accumulation in the concentrate, which occurs more slowly than the TSC decrease in dialysate. This is driven by various speed or selectivity of the electrolyte component transmission through the membranes. It is obvious that the difference in the rates of substance transfer from the dialysate line to the concentrate line is the basis for the electromembrane separation of complex multicomponent solutions.

With a maximum current of $28.3 \mathrm{~A}$ and a flow rate of working solutions of $1500 \mathrm{dm}^{3} / \mathrm{h}$ in one pass, the TSC in the concentrate rises by about $4 \mathrm{~g} / 1$ in terms of $100 \%$ $\mathrm{NaOH}$ or $0.4 \%$ mass. The efficiency drop of the electrodialysis process begins when the dialysate salinity is less than $0.2 \mathrm{~g} / 1$ and sharply decreases when the salinity is less than $0.1 \mathrm{~g} / \mathrm{l}$. Power consumption is on average $1.0 \mathrm{kWh} / \mathrm{kg}$.

To determine the selectivity and the dialysis rate of the components, the total material balance for dialysate and concentrate lines was calculated. The separation ratio determines the proportions of the electrolyte components in the concentrate. The concentration ratio shows the ratio of the electrolyte component accumulation in the concentrate relative to each other per unit of time. Using the reduction ratio, it becomes possible to establish the rate of electrolyte component transition from the dialysate line. The specific energy consumption of dialysis characterizes the amount of energy required for the electrolyte matter transfer from the dialysate line to the concentrate line per unit of time per unit volume.
Figure 2 shows the curves of electrolyte component accumulation in a concentrate line as a function of time and electricity passed through the apparatus.

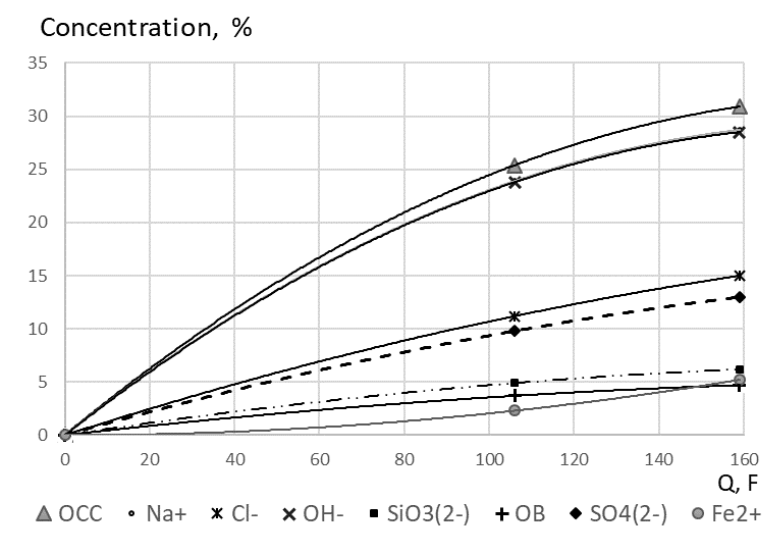

Figure 2. Material balance dynamic of components in the concentrate line (\% of the initial content in dialysate) during electrodialysis of fraction 4 .

According to the results of alkaline fractions electrodialysis of anion-exchange filter regeneration solutions, we detected significant differences in the rate or selectivity of the electrolyte component transition from the dialysate line to the concentrate line through the membranes. According to the selectivity of transfer through membrane pairs from dialysate relative to total salt content, the main components are arranged in a row:

$$
\mathrm{OH}^{-} \approx \mathrm{Na}^{+}>\mathrm{SO}_{4}{ }^{2-} \approx \mathrm{Cl}^{-}>\mathrm{Hum}^{-} \approx \mathrm{SiO}_{3}{ }^{2-} \approx \mathrm{Fe}^{2+}
$$

High transfer selectivity is shown by hydroxide ions and sodium ions. Chloride ions and sulfates are in an intermediate position, and silicates, organic substances and iron ions are poorly transported in an electric field, or, judging by the reduction factor, are retained by the membranes. This means that alkali obtained in the concentrate line is cleaner for the listed impurities than the original one after regeneration. For 1.5 hours of AWRS electrodialysis the alkaline solution has twice less amount of chlorides and sulfates and 5-6 times less 
amount of silicates, organic substances and iron as compared with transferred alkali.

\section{Conclusion}

1. After regeneration of anion exchange filters, the original alkaline solution in the amount of $50 \%$ by weight is sent for neutralization, that is, in wastewater. It is necessary to effectively use the alkali discharged into drains.

2. Electro-membrane technologies allow separating complex multicomponent highly concentrated solutions.

3. Electrodialysis of alkaline waste regeneration solutions and wash water after regeneration of anionite filters at a water treatment plant was carried out. Significant differences in the selectivity of the electrolyte component transition from the separable solution through the membranes and, consequently, in the rate of their accumulation in the resulting alkaline concentrate were detected.

4. Depending on time and amount of transmitted electricity, it is possible to obtain an alkaline solution purified from impurities from multicomponent alkaline spent regeneration solutions and wash water after regeneration of anion-exchange filters of a water treatment plant

\section{References}

[1] Y.N. Zvonareva, Y.V. Vankov, A.A. Antonov, V.G. Mikhaylenko, Purification Of Industrial Effluents By The Electro-Membrane Softening Method III International Youth Scientific Conference 17-19 (2015)

[2] D.N. Volodin, N.V. Magomedova, A.N. Voropaev, The Use Of Electro-Membrane Technologies In Wastewater Treatment Water Treatment. Water Supply 8, 32-36 (2015)

[3] A.A. Tarelin, V.G. Mikhaylenko, A.E. Khinevich, E.F. Lukyanov, O.I. Knyazeva, T.S. Vitkovskaya, A.V. Antonov, Influence Of Feedwater Impurities On The Reliability Of Boiler-Turbine Equipment Modern Science: Research, Ideas, Results, Technologies 2, 92-98 (2013)

[4] V.G. Mikhaylenko, A.V. Antonov, O.I. Lukyanova, T.A. Berezka, The Possibility Of Conducting At The TPP Plant An Electro-Membrane Treatment Of Network Water For Its Softening Thermal Engineering 12, 78-83 (2018)

[5] S.I. Niftaliev, Et Al Application Of Electrodialysis To Obtain Acid And Alkali From A Concentrated Solution Of Sodium Sulfate VGUIT 4 175-179 (2014)

[6] F. Ferella, I.De. Michelis, F. Veglio, Process For The Recycling Of Alkaline And Zinc-Carbon Spent Batteries Journal Of Power Sourses 1, 78-82 (2008)

[7] N. Zhang, High electrodialysis - high pressure that contains salt industrial waste water is received and is strained combination branch salt system Patent CN205528158U (2016)
[8] D. Valero, Application Of Electrodialysis For The Treatment Of Almond Industry Wastewater Journal Of Membrane Science 476, 580-589 (2015)

[9] S. Hosimin, Four compartment mono selective electrodialysis for separation of sodium formate from industry wastewater Chemical Engineering Journal 333, 162-169 (2018)

[10] W.S. Fernanda, Treatment of Effluent from the Agate Dyeing Industry Using Photodegradation and Electrodialysis Processes Separation Science and Technology 50, 142-147 (2015)

[11] M. Wang, The Reclamation Of Brine Generated From Desalination Process By Bipolar Membrane Electrodialysis Journal Of Membrane Science 452, 5461 (2014)

[12] P. Krizhanek, L. Novak, The Future Of ElectroMembrane Processes Industrial Production And Use Of Elastomers 3, 30-39 (2016)

[13] A. Doyen, C. Udenigwe, P. Mitchell, A. Marette, R. Aluko, L. Bazinet, Anti-Diabetic And Antihypertensive Activities Of Two Flaxseed Protein Hydrolysate Fractions Revealed Following Their Simultaneous Separation By Electrodialysis With Ultrafiltration Membranes Food Chemistry 145, 66-76 (2014)

[14] R. He, A. Girgih, E. Rozoy, L. Bazinet, X. Ju, R. Aluko, Selective Separation And Concentration Of Antihypertensive Peptides From Rapeseed Protein Hydrolysate By Electro-Dialysis With Ultrafiltration Membranes Food Chemistry 197, 1008-1014 (2016)

[15] S. Suwal, C. Roblet, A. Doyen, J. Amiot, L. Beaulieu, J. Legault, L. Bazinet, Electrodialytic Separation Of Peptides From Snow Crab By-Product Hydrolysate: Effect Of Cell Configuration On Peptide Selectivity And Local Electric Field Separation And Purification Technology 127, 29-38 (2014)

[16] Y. Haiyang, X. Chunyan, L. Wei, W. Yaoming, $\mathrm{X}$. Tongwen, Electrodialysis To Concentrate Waste Ionic Liquids: Optimization of Operating Parameters Ind Eng Chem Res 55, 2144-2152 (2016)

[17] N.D. Chichirova, A.A. Chichirov, A.I. Lyapin, A.I. Minibaev, I.Yu. Silov, L.I. Tolmachev, Electromembrane Utilization Of Highly Mineralized Alkaline Purge Waters of The Evaporative Water Treatment Plant Of Thermal Power Plants Reliability And Safety Of Power Engineering 4, 41-45 (2015) 